\title{
Quantum signatures of charge flipping vortices in the Bose-Hubbard trimer
}

\author{
Peter Jason and Magnus Johansson* \\ Department of Physics, Chemistry and Biology (IFM), Linköping University, SE-581 83 Linköping, Sweden
}

(Received 20 June 2016; published 15 November 2016)

\begin{abstract}
In this work we study quantum signatures of charge flipping vortices, found in the classical discrete nonlinear Schrödinger trimer, by use of the Bose-Hubbard model. We are able to identify such signatures in the quantum energy eigenstates, for instance when comparing the site amplitudes of the classical charge flipping vortices with the probability distribution over different particle configurations. It is also discussed how to construct quantum states that correspond to the classical charge flipping vortices and which effects can lead to deviations between the classical and quantum dynamics. We also examine properties of certain coherent states: classical-like quantum states that can be used to derive the classical model. Several quantum signatures are identified when studying the dynamics of these coherent states, for example, when comparing the average number of particles on a site with the classical site amplitude, when comparing the quantum and classical currents and topological charge, and when studying the evolution of the quantum probability amplitudes. The flipping of the quantum currents are found to be an especially robust feature of these states.
\end{abstract}

DOI: 10.1103/PhysRevE.94.052215

\section{INTRODUCTION}

The Bose-Hubbard model has become a prominent and much studied model in physics through the study of BoseEinstein condensates in optical lattices. This has been a very active and prolific area of research in the past few decades and is among other things considered a promising candidate for quantum computers and simulators $[1,2]$. In the classical limit of the Bose-Hubbard model, corresponding to a large number of bosons per site, it is well approximated by the discrete nonlinear Schrödinger (DNLS) model [3,4]. The DNLS model is one of the most studied nonlinear lattice models and has, apart from Bose-Einstein condensates, also applications for, e.g., optical wave-guide systems [5].

Of fundamental interest here is the quantum-classical correspondence, and especially how structures connected to the nonlinearity of the classical DNLS model emerge from the inherently linear quantum model. Studies of this kind are numerous and include those of discrete breathers $[4,6]$, compactons [7,8], and vortices [9], as well as the self-trapping transition [10,11], modulational instabilities [12,13], and oscillatory instabilities [14]. More recently, Ref. [15] studied quantum signatures of Arnold diffusion in Bose-Hubbard lattices with few sites (especially three and four sites).

The Hilbert space of the Bose-Hubbard model increases very rapidly with the number of sites and particles, and one is typically limited to quite modest system sizes, especially when using exact diagonalization. One beneficial aspect of studying small lattices is therefore that it enables us to use (in this context) many particles and better connect the classical and quantum world. Smaller lattices are also experimentally accessible nowadays, for instance, by painting optical potentials [16].

We will in this paper look for quantum signatures of the charge flipping vortices (CFVs) recently found and studied in the DNLS trimer (symmetric triangular configuration)

\footnotetext{
*mjn@ifm.liu.se; https://people.ifm.liu.se/majoh
}

[17]. A vortex is the rotational flow of a field around a topological phase dislocation; a point where the field amplitude vanishes and around which the field changes its phase by an integer multiple of $2 \pi$. This integer is the topological charge of the vortex, and its sign indicating the vortex' direction. CFVs are characterized by a repeated flipping of the topological charge, and consequently also a reversal of the flow. The topological charge is conserved in systems with full rotational symmetry, which thus needs to be broken to enable CFVs, for instance, by introducing an underlying lattice potential. CFVs have been studied in a wide variety of lattice geometries and configurations, both theoretically [17-22] and experimentally in photonic lattices [23-25]. The CFVs found in Ref. [17] are periodic solutions (in corotating frames) that exist in families that can be followed continuously between two stationary solutions: the single depleted well (SDW) solution, $\psi=\left(\psi_{1},-\psi_{1}, 0\right)$, and a dimerlike solution $\psi=\left(\psi_{1},-\psi_{2},-\psi_{2}\right) ; \psi_{1}, \psi_{2} \in \mathbb{R}^{+}\left(\psi_{1}>\psi_{2}\right)$ [26]. The topological charge of these CFVs will naturally have the same periodicity as the CFVs themselves.

The dimerlike solution is the "discrete breather" ground state, with a peak on one site [27].

The outline of the paper is as follows. Section II contains some theoretical background, with Sec. II A introducing the classical and quantum model, Sec. II B discussing some aspects of coherent states, and Sec. IIC describing how to calculate classical and quantum currents and topological charge. Section III begins by recapitulating some results for the classical CFVs, before examining CFV quantum signatures in the energy eigenstates. Section IV focuses on the classicallike coherent states, with Sec. IV A examining the quantum dynamics of these, Sec. IV B studying their projection onto energy eigenstates, and Sec. IV C analyzing how the quantum dynamics changes when using classical CFVs at different times of their evolution as input to the coherent states. The quantum results in Secs. III and IV are for a specific number of particles and values of the nonlinear parameter, and Sec. V discusses some effects of changing these. Section VI finishes the paper with a summary and conclusions. 


\section{THEORY}

\section{A. Models}

Consider the Bose-Hubbard trimer on the following form:

$$
\hat{H}=\sum_{j=1}^{3}\left[\hat{a}_{j+1}^{\dagger} \hat{a}_{j}+\hat{a}_{j}^{\dagger} \hat{a}_{j+1}-\frac{\alpha}{2} \hat{n}_{j}\left(\hat{n}_{j}-1\right)\right],
$$

where $\hat{a}_{j}^{(\dagger)}$ is the bosonic annihilation (creation) operator for site $j, \hat{n}_{j}=\hat{a}_{j}^{\dagger} \hat{a}_{j}$ the number operator for the same site, and $\alpha>0$ determines the interaction strength. Because of the periodicity of the lattice $\hat{a}_{4}^{(\dagger)}=\hat{a}_{1}^{(\dagger)}$. This differs from the more standard form of the Bose-Hubbard model with repulsive interactions:

$$
\hat{H}^{\prime}=\sum_{j=1}^{3}\left[-J\left(\hat{a}_{j+1}^{\dagger} \hat{a}_{j}+\hat{a}_{j}^{\dagger} \hat{a}_{j+1}\right)+\frac{U}{2} \hat{n}_{j}\left(\hat{n}_{j}-1\right)\right],
$$

where $J>0$ and $U>0$ are the hopping and (repulsive) interaction parameter, respectively. The two Hamiltonians are, however, related by $\hat{H}=-\hat{H}^{\prime} / J$ and $\alpha=U / J$, which not only rescales the energies but also flips the energy spectrum. We have chosen to use Hamiltonian Eq. (1) to facilitate comparisons with the classical results in Ref. [17]. We will also deal with the lowest eigenstates of Hamiltonian Eq. (1), which correspond to the highest eigenstates of the standard Hamiltonian Eq. (2).

The quantum states of the Bose-Hubbard model are expressed with Fock states, $\left|n_{1}, n_{2}, n_{3}\right\rangle$, where $n_{j} \geqslant 0$ is the number of bosons on site $j$. Because Hamiltonian Eq. (1) commutes with the total number operator $\hat{N}=\sum_{j=1}^{3} \hat{n}_{j}$, its eigenstates have a specified total number of particles $N=n_{1}+n_{2}+n_{3}$, and we will therefore always work in subspaces with a fixed $N$. This implies that $n_{j}$ for only two sites need to be specified for a Fock state, and that the probability distribution of the different particle configurations $\left(n_{1}, n_{2}, n_{3}\right)$ may conveniently be illustrated with two-dimensional triangular plots, similar as in Refs. [11,15]. We will frequently use the relative number of particles per site, $v_{j}=n_{j} / N$, and the associated operator $\hat{v}_{j}=\hat{n}_{j} / N$, since it simplifies the comparison of systems with different $N$.

Furthermore, both $\hat{H}$ and $\hat{N}$ commute with the translation operator $\hat{T}\left|n_{1}, n_{2}, n_{3}\right\rangle=\left|n_{3}, n_{1}, n_{2}\right\rangle$. The energy eigenstates will therefore also be labeled with a $k$ value, which is related to the eigenvalues of $\hat{T}$ by $\hat{T}|\psi\rangle=e^{i k}|\psi\rangle$, and may take the values $k=0, \pm 2 \pi / 3$ for the trimer. We will denote the $m$ th lowest energy in subspace $k$, and the associated eigenstate, with $E_{m}(k)$ and $\left|E_{m}(k)\right\rangle$. Since there is no preferred direction in the model, the spectra for $k= \pm 2 \pi / 3$ are identical, and the \pm -sign is hereafter omitted.

In the classical limit of a large number of particles, the BoseHubbard model is well approximated by the DNLS model. Formally, this can be derived by using the "time-dependent variational principle" (TDVP) with SU(3) coherent states as trial states [generally, $\mathrm{SU}(f)$ coherent states for $f$ number of sites] [28]. The SU(3) coherent states are defined as

$$
\begin{gathered}
|C[\psi]\rangle=\frac{1}{\sqrt{N !}}\left(\sum_{j=1}^{3} \psi_{j} \hat{a}_{j}^{\dagger}\right)^{N}|\mathrm{vac}\rangle, \\
\mathcal{N}=\sum_{1}^{3}\left|\psi_{j}\right|^{2}=1,
\end{gathered}
$$

$\mid$ vac $\rangle$ being the vacuum state. Other trial states can also be used, e.g., the Glauber coherent states familiar from quantum optics $[29,30]$, but the advantage of the SU(3) coherent states is that they have a fixed total number of particles $N$ [28]. Note that we let the classical state vector $\psi=\left(\psi_{1}, \psi_{2}, \psi_{3}\right)$ be normalized to one, unlike in Ref. [28] where it is normalized to $N$ (see Ref. [8] for further discussions). The actual quantum state is, however, the same. We will consider two different time-evolved states, which we will denote $|C[\psi(t)]\rangle$ and $\left|C_{0}[\psi](t)\right\rangle$, respectively. The state $|C[\psi(t)]\rangle$ corresponds to $\psi(t)$ inserted into Eq. (3), where $\psi(t)$ has been obtained by integrating the DNLS Eq. (5) with the initial condition $\psi(0) .\left|C_{0}[\psi](t)\right\rangle$, on the other hand, is the quantum time evolution, calculated with the Bose-Hubbard Hamiltonian Eq. (1), of the coherent state obtained when inserting $\psi=\psi(0)$ into Eq. (3). Note that the quantum time evolution of an initially coherent state generally does not produce another coherent state, as is emphasized by the state's subscript.

Using SU(3) coherent states in the TDVP means that $\langle C[\psi]|i \partial / \partial t-\hat{H}| C[\psi]\rangle=0$, leading to

$$
i \dot{\psi}_{j}-\left(\psi_{j-1}+\psi_{j+1}\right)+\underbrace{\alpha(N-1)}_{\beta}\left|\psi_{j}\right|^{2} \psi_{j}=0,
$$

which we identify as the DNLS model with nonlinear parameter $\beta=\alpha(N-1)$ [5]. Equation (5) indicates that $\alpha$ must scale as $\sim N^{-1}$ with the number of particles for the DNLS to be the proper classical limit. The classical Hamiltonian associated with Eq. (5) is

$$
\mathcal{H}=\sum_{j=1}^{3}\left(\psi_{j} \psi_{j+1}^{*}+\psi_{j+1} \psi_{j}^{*}-\frac{\beta}{2}\left|\psi_{j}\right|^{4}\right),
$$

with $\left\{\psi_{j}\right\}$ and $\left\{i \psi_{j}^{*}\right\}$ as canonical coordinates and momenta, respectively. By scaling $\alpha \sim N^{-1}$, the eigenvalues of the Hamiltonian Eq. (1) scale as $\sim N$. For a classical state with energy $\mathcal{H}$, the energy of a corresponding eigenstate can be approximated by $N \mathcal{H}$.

Reference [17] uses a DNLS model like Eq. (5) but with $\beta$ fixed to unity and a variable norm $\mathcal{N}=\sum_{j=1}^{3}\left|\psi_{j}\right|^{2}$. Rescaling $\psi$ shows that this is equivalent to Eq. (5) (i.e., with normalized $\psi$ ) with the nonlinear parameter $\beta=\mathcal{N}$. The norm of a CFV in Ref. [17] will thus be the nonlinearity for the corresponding normalized CFV in Eq. (5), and we will only use the latter type in the rest of the paper.

\section{B. Coherent states}

Coherent states are generally thought to be the quantum states that best describe classical systems, and they have several useful properties to this end [31]. For instance, the 
input vector $\psi$ of a coherent state Eq. (3) becomes the dynamical variable in the classical description Eq. (5), and there is thus a one-to-one correspondence between classical states and coherent states. Another important property is that $\left\langle C[\psi]\left|\hat{v}_{j}\right| C[\psi]\right\rangle=\left|\psi_{j}\right|^{2}$. From Bohr's correspondence principle we expect that $\left\langle C_{0}[\psi](t)\left|\hat{v}_{j}\right| C_{0}[\psi](t)\right\rangle \rightarrow\left|\psi_{j}(t)\right|^{2}$ in the limit $N \rightarrow \infty$, while the variance of $\hat{v}_{j}$ approaches zero. We will examine how well $\left\langle C_{0}[\psi](t)\left|\hat{v}_{j}\right| C_{0}[\psi](t)\right\rangle$ follows $\left|\psi_{j}(t)\right|^{2}$ for finite $N$ in different regimes, and how the classical dynamics emerges from the quantum states. Consider, therefore,

$$
\begin{aligned}
\left\langle\hat{v}_{j}\right\rangle= & \left\langle C_{0}[\psi](t)\left|\hat{v}_{j}\right| C_{0}[\psi](t)\right\rangle \\
= & \sum_{k, k^{\prime}, m, m^{\prime}} d_{m}(k)\left(d_{m^{\prime}}\left(k^{\prime}\right)\right)^{*}\left\langle E_{m^{\prime}}\left(k^{\prime}\right)\left|\hat{v}_{j}\right| E_{m}(k)\right\rangle \\
& \quad \times \exp \left(-i\left[E_{m}(k)-E_{m^{\prime}}\left(k^{\prime}\right)\right] t\right),
\end{aligned}
$$

where $\left|C_{0}[\psi](0)\right\rangle=\sum_{k, m} d_{m}(k)\left|E_{m}(k)\right\rangle(\hbar=1)$. The time evolution of $\left\langle\hat{v}_{j}\right\rangle$ in Eq. (7) is (like any observable) determined by the structure of the occupied eigenstates and how their energies are related. Deviations between $\left\langle\hat{v}_{j}\right\rangle$ and $\left|\psi_{j}\right|^{2}$ can, as we will see later on, be associated with the structure of the eigenstates and energy spectrum.

\section{Topological charge}

A continuity equation can be obtained for the classical site amplitudes from Eq. (5),

$$
\frac{d\left|\psi_{j}\right|^{2}}{d t}=\mathcal{J}_{j-1}-\mathcal{J}_{j},
$$

where

$$
\mathcal{J}_{j}=-2 \Im\left(\psi_{j}^{*} \psi_{j+1}\right)=-2\left|\psi_{j}\right|\left|\psi_{j+1}\right| \sin \left(\theta_{j+1}-\theta_{j}\right)
$$

gives the current from site $j$ to $j+1$, with $\psi_{j}=\left|\psi_{j}\right| e^{i \theta_{j}}$, $-\pi<\theta_{j+1}-\theta_{j} \leqslant \pi$. Related to this is the topological charge $\tau$, which for classical vortices indicates the direction of the energy flow. The $\tau$ is for the DNLS trimer given by

$$
\tau=\frac{1}{2 \pi} \sum_{j=1}^{3} \arg \left(\psi_{j}^{*} \psi_{j+1}\right)=\frac{1}{2 \pi} \sum_{j=1}^{3}\left(\theta_{j+1}-\theta_{j}\right),
$$

which is restricted to $0, \pm 1$ for the trimer. Turning to quantum mechanics, we can derive a corresponding continuity equation for $\hat{n}_{j}$ by using the Heisenberg equation, giving

$$
\frac{d \hat{n}_{j}}{d t}=\hat{J}_{j-1}-\hat{J}_{j}
$$

with

$$
\hat{J}_{j}=-i\left(\hat{a}_{j+1}^{\dagger} \hat{a}_{j}-\hat{a}_{j+1} \hat{a}_{j}^{\dagger}\right)
$$

being the current operator from site $j$ to $j+1$. Comparing Eqs. (9) and (12) hints how we may calculate the quantum phase difference between sites, denoted as $\theta_{j+1}^{(Q M)}-\theta_{j}^{(Q M)}$, and thus the quantum topological charge (see Ref. [32] for discussions and definitions of quantum phase operators). We will calculate the average value of the phase difference between two sites with [9]

$$
\begin{aligned}
\left\langle\cos \left(\theta_{j+1}^{(Q M)}-\theta_{j}^{(Q M)}\right)\right\rangle & =\frac{\left\langle\hat{a}_{j+1}^{\dagger} \hat{a}_{j}+\hat{a}_{j+1} \hat{a}_{j}^{\dagger}\right\rangle}{\sqrt{2\left\langle 2 \hat{n}_{j} \hat{n}_{j+1}+\hat{n}_{j}+\hat{n}_{j+1}\right\rangle}}, \\
\left\langle\sin \left(\theta_{j+1}^{(Q M)}-\theta_{j}^{(Q M)}\right)\right\rangle & =\frac{\left\langle i\left(\hat{a}_{j+1}^{\dagger} \hat{a}_{j}-\hat{a}_{j+1} \hat{a}_{j}^{\dagger}\right)\right\rangle}{\sqrt{2\left\langle 2 \hat{n}_{j} \hat{n}_{j+1}+\hat{n}_{j}+\hat{n}_{j+1}\right\rangle}} .
\end{aligned}
$$

This will in practice be done by taking arcsin of Eq. (13b), with the range determined from the expectation value of the cosine function Eq. (13a). Replacing $\theta_{j+1}-\theta_{j}$ with $\left\langle\theta_{j+1}^{(Q M)}-\theta_{j}^{(Q M)}\right\rangle$ in Eq. (10) gives the formula used for the quantum topological charge. Due to quantum fluctuations in the phase operators Eq. (13), the quantum topological charge is not restricted to integer values, unlike the classical topological charge (compare with the melting of stationary vortices studied in Ref. [9]).

\section{QUANTUM SIGNATURES IN EIGENSTATES}

In this section we look for signatures of the classical CFVs in the energy eigenstates, and let us therefore begin by recapitulating some results for the classical CFVs [17]. The CFVs are periodic solutions (in a corotating frame) with period $T$, that exist in continuous families, which can be followed between the stationary dimerlike solution and the stationary SDW solution. We therefore introduce the relative Hamiltonian for the CFVs:

$$
\Delta \mathcal{H}_{\text {rel }}=\left(\mathcal{H}_{\mathrm{CFV}}-\mathcal{H}_{\text {dimerlike }}\right) /\left(\mathcal{H}_{\text {SDW }}-\mathcal{H}_{\text {dimerlike }}\right),
$$

with the Hamiltonian Eq. (6) calculated for the same nonlinear parameter $\beta$, and the subscript of $\mathcal{H}$ indicates the solution for which it is calculated. The left column of Fig. 1 shows $\left|\psi_{j}(t)\right|^{2}$ of four CFVs that belong to the same subfamily for $\beta=1.00$. The initial conditions are chosen so that $\left|\psi_{1}\right|^{2}$ and $\left|\psi_{3}\right|^{2}$ have minima, while $\left|\psi_{2}\right|^{2}$ has a maximum, at $t=0$. Close to the dimerlike solution, the CFVs perform small oscillations in $\left|\psi_{j}(t)\right|^{2}$ [cf. Fig. 1(a1)], which essentially corresponds to a perturbation of the linearly stable dimerlike solution. By following the CFV subfamily toward the SDW solutions (going down along the left column of Fig. 1), the oscillations in $\left|\psi_{j}(t)\right|^{2}$ increase, so that close to the SDW solution, the CFVs essentially consist of a "hole" (an empty site) that goes back and forth between two sites [cf. Fig. 1(d1)]. These CFVs can be viewed as the result of perturbing the linearly unstable SDW solution. The SDW solution is linearly unstable, through a nonoscillatory instability, for all positive values of $\alpha$ in Eq. (5). This is in contrast to when $\alpha$ is negative, where the SDW solution becomes oscillatory unstable in a parameter window but is linearly stable otherwise [33]. Note that the initial conditions in the left column of Fig. 1 are chosen to be as close as possible to a SDW in this limit.

A different perspective on the classical CFVs is given in terms of localized discrete breathers. The dimerlike solution is then the on-site centered breather ground state, while the SDW solution is an intersite centered breather. The quantity $\mathcal{H}_{\text {SDW }}-\mathcal{H}_{\text {dimerlike }}$ is interpreted as the Peierls-Nabarro (PN) potential barrier that needs to be overcome to transfer the on-site breather to its neighbor site [34]. The CFVs can thus 

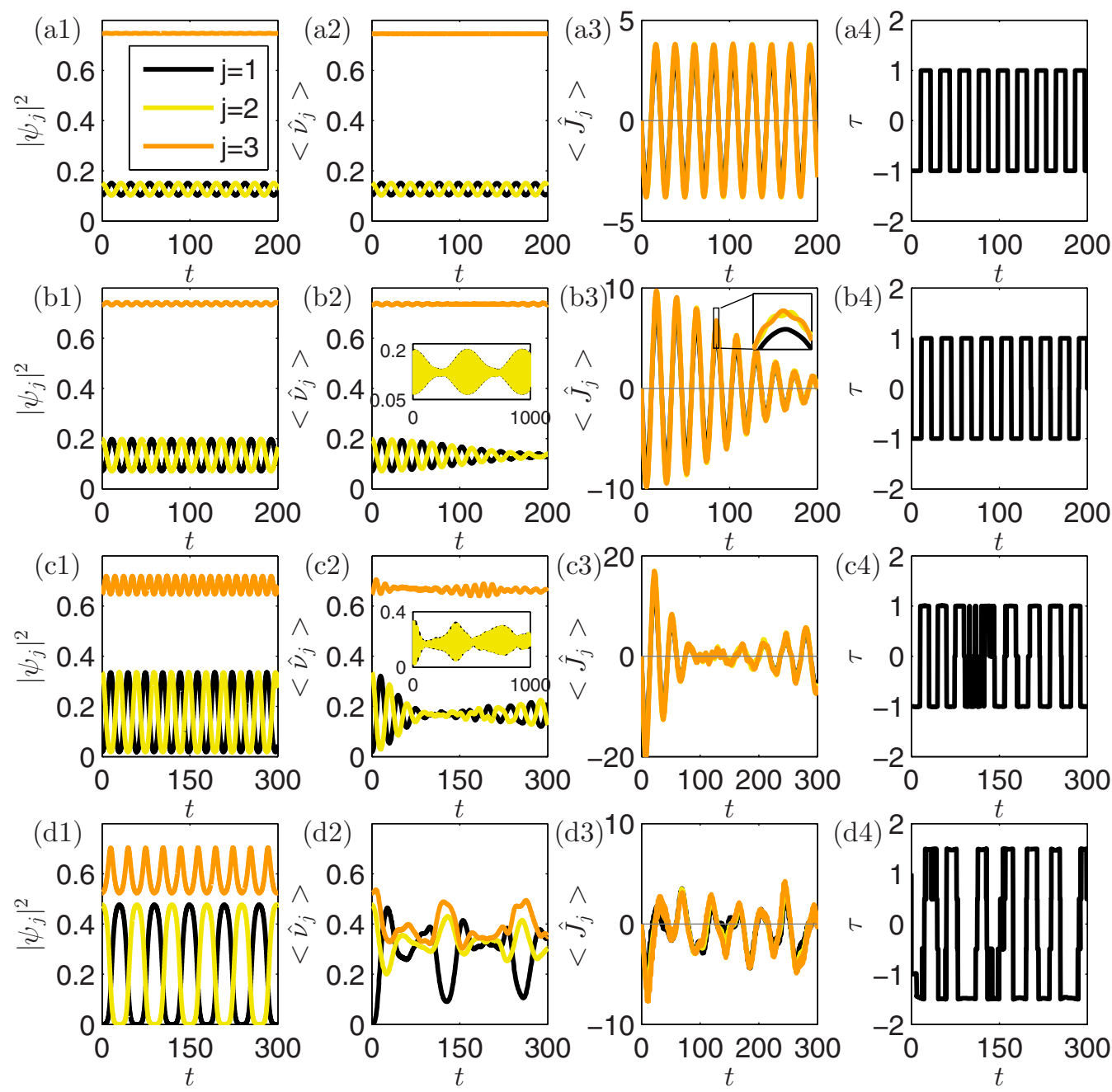

FIG. 1. First column: $\left|\psi_{j}(t)\right|^{2}$ for classical CFVs with $\beta=1.00$. From top to bottom: $\Delta \mathcal{H}_{\text {rel }}=0.024,0.141,0.670$, and 0.996. Second column: time evolution of $\left\langle\hat{v}_{j}\right\rangle=\left\langle C_{0}[\psi](t)\left|\hat{v}_{j}\right| C_{0}[\psi](t)\right\rangle$ for CFV coherent states with $\psi=\psi(0)$ of the classical CFV on the same row, $N=100$ and $\alpha=\beta /(N-1)=1 / 99$. The insets show $\left\langle\hat{v}_{j}\right\rangle$ over a longer time. Third column: average currents $\left\langle\hat{J}_{j}\right\rangle$. Fourth column: quantum topological charge $\tau$. Both the currents and topological charge are calculated from the CFV coherent state on the same row. $\left\langle\hat{J}_{2}\right\rangle$ follows $\left\langle\hat{J}_{3}\right\rangle$ quite closely and is hard to distinguish in the plots.

be considered to be breather states that do not overcome the PN-barrier $\left(\Delta \mathcal{H}_{\text {rel }}<1\right)$, but instead oscillate back and forth in the PN potential around the minimum of the on-site centered ground state. Let us point out that Ref. [26] uses a DNLS model with opposite signs on both the neighbor coupling and nonlinear parameter, meaning that the dimerlike solution (on-site centered breather) is the highest eigenstate rather than the ground state. A corresponding single-peaked solution exists also for the DNLS trimer with opposite sign only on the coupling. This solution exists above a certain threshold value for the nonlinear parameter, where it bifurcates from another solution [27], and it also becomes the ground state above another parameter value [35].

Consider now Fig. 2(a), which plots the trajectories of some classical solutions from the same subfamily as in the left column of Fig. 1. The black triangle is the border of the accessible area defined by $\sum_{j}\left|\psi_{j}\right|^{2}=1$. The black crosses mark the dimerlike solutions and the black circles the SDW solutions, while the orange (dark gray) and yellow (light gray) curves are the same CFVs as in Figs. 1(b1) and 1(d1), respectively. The CFVs are thus represented as arcs in Fig. 2(a), with CFVs close to the dimerlike solution being small arcs close to the black cross, similar to the orange (dark gray) one. Moving along the CFVs subfamily toward the SDW solution means that the arc grows, until it touches the two circles of the SDW solution, similar to the yellow (light gray) arc. A larger arc thus corresponds both to larger oscillations in $\left|\psi_{j}\right|^{2}$ and to a larger energy (Hamiltonian). In this discussion, we have assumed that the third site is the most occupied, but the CFVs can be translated in the lattice, corresponding essentially to a rotation of the triangle.

Turning now to the quantum eigenstates, Figs. 2(b)-2(f) display $\left|\left\langle n_{1}, n_{2}, n_{3} \mid E_{m}(0)\right\rangle\right|^{2} / \max _{n_{1}, n_{2}, n_{3}}\left(\left|\left\langle n_{1}, n_{2}, n_{3} \mid E_{m}(0)\right\rangle\right|^{2}\right)$ for $m=1,2,3,7,11$ with $N=100$ and $\alpha=\beta /(N-1)=$ $1 / 99$, with the energy increasing from Fig. 2(b) to 2(f). Figure 2(g) shows $\left|\left\langle n_{1}, n_{2}, n_{3} \mid E_{11}(2 \pi / 3)\right\rangle\right|^{2} / \max _{n_{1}, n_{2}, n_{3}}$ $\left(\left|\left\langle n_{1}, n_{2}, n_{3} \mid E_{11}(2 \pi / 3)\right\rangle\right|^{2}\right)$ for the same $N$ and $\alpha$. As can be seen in these plots, the eigenstates spread out more over the 

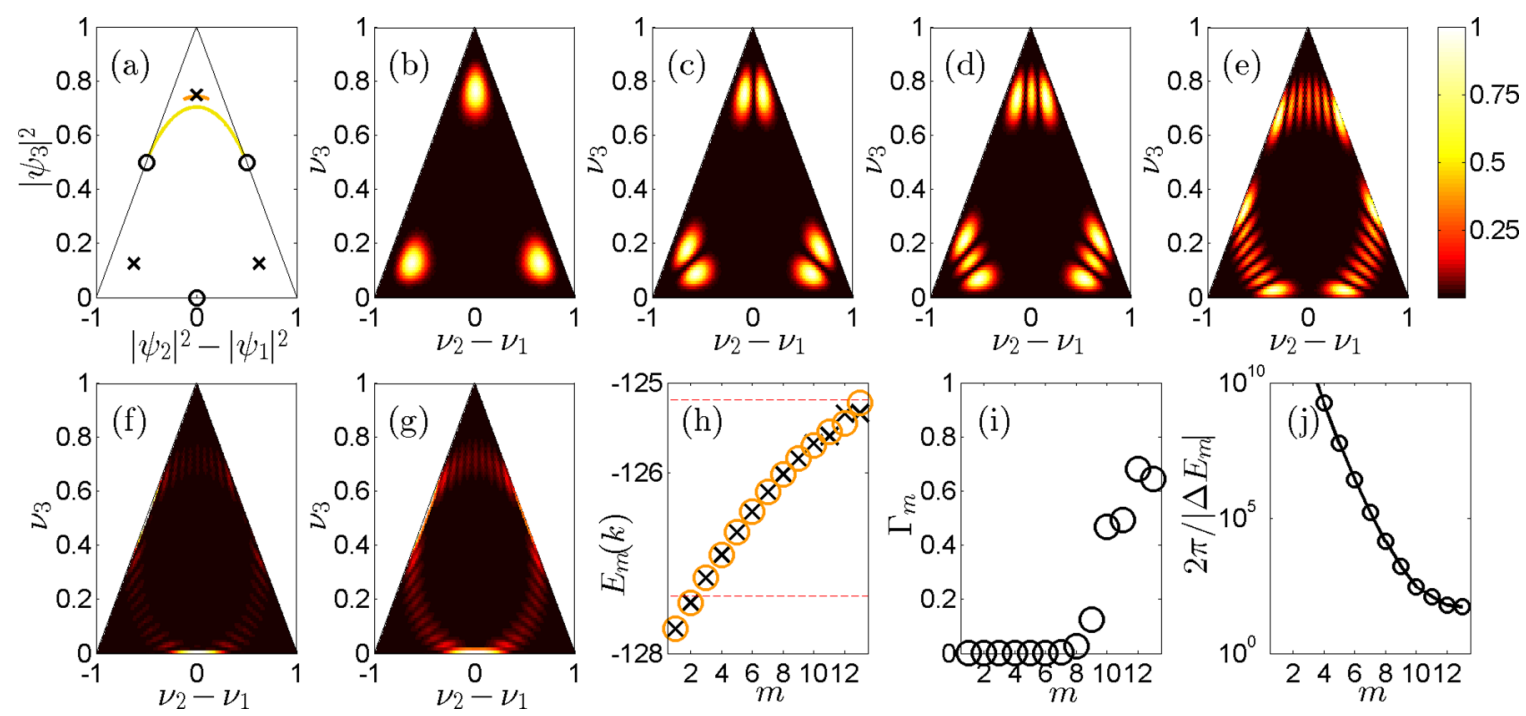

FIG. 2. (a) Trajectories of classical solutions for $\beta=1.00$. The black crosses mark dimerlike solutions while the black circles mark the SDW solutions. The orange (dark gray) and yellow (light gray) lines are the CFVs of Figs. 1(b1) and 1(d1), respectively. For (b)-(j) $N=100$ and $\alpha=\beta /(N-1)=1 / 99$. (b)-(g) $\left|\left\langle n_{1}, n_{2}, n_{3} \mid E_{m}(k)\right\rangle\right|^{2} / \max _{n_{1}, n_{2}, n_{3}}\left(\left|\left\langle n_{1}, n_{2}, n_{3} \mid E_{m}(k)\right\rangle\right|^{2}\right)$, where (b)-(f) $k=0$ and $m=1,2,3,7,11[$ energy increasing from (b) to (f)], and (g) $k=2 \pi / 3$ and $m=11$. (h) Lower part of the energy spectrum for $k=0$ (crosses) and $k=2 \pi / 3$ (circles). $N \mathcal{H}_{\text {SDW }}$ and $N \mathcal{H}_{\text {dimerlike }}$ are include as the top and bottom dashed lines, respectively. (i) $\Gamma_{m}$ of Eq. (15). (j) $2 \pi /\left|E_{m}(0)-E_{m}(2 \pi / 3)\right|$.

triangle when increasing the energy. The size of the bright lobes in the plots indicate the quantum uncertainty of $v_{j}$, which shrinks as $N$ is increased. Figure 2(h) shows the energy spectrum of the 13 lowest eigenstates in each $k$ space, with $N \mathcal{H}_{\text {dimerlike }}$ and $N \mathcal{H}_{\text {SDW }}$ included as dashed, red lines. The line for the dimerlike solution does not coincide with the ground state, but it should approach it as $N$ is increased further. The region between the dashed lines indicates (approximately) which eigenstates that will contribute to the quantum CFVs.

Due to the Bloch theorem, all eigenstates have a translational invariance, which is manifested in the similarity of the triangles in Figs. $2(\mathrm{~b})-2(\mathrm{~g})$ when rotated $\pm 2 \pi / 3$. More classical-like (symmetry-broken) states can be obtained by adding together eigenstates from different $k$ spaces with suitable coefficients. The basic idea is to cancel out certain bright lobes in the probability distribution plots, leaving them only in one third of the triangle. In order for this to work well, it is necessary that we combine eigenstates with similar occupation of Fock states, i.e., similar triangular probabilitydistribution plots. A plot corresponding to Fig. 2 for $k=2 \pi / 3$ would reveal that the $m$ th lowest eigenstates in each $k$ space look similar, especially for low $m$. As a quantitative measure of how similar they are, we introduce

$$
\begin{aligned}
\Gamma_{m}= & \left.\sum_{n_{1}, n_{2}, n_{3}}||\left\langle n_{1}, n_{2}, n_{3} \mid E_{m}(0)\right\rangle\right|^{2} \\
& -\left|\left\langle n_{1}, n_{2}, n_{3} \mid E_{m}(2 \pi / 3)\right\rangle\right|^{2} \mid .
\end{aligned}
$$

Note that this quantity completely disregards the phase between the Fock states. This quantity is shown for $m=$ $1, \ldots, 13$ in Fig. 2(i), and $\Gamma_{m}$ is very small for $m<9$, but increases for larger $m$. Figures 2(f) and 2(g) are for $m=11$, and $k=0$ and $k=2 \pi / 3$, respectively, and it is evident that these plots differ quite significantly. Note also that the larger $\Gamma_{m}$ for $m>9$ is associated with an increased energy splitting in Fig. 2(j). The "lifetime" of the localization, obtained from the $m$ th lowest eigenstates, is associated with the inverse of the energy splitting $\Delta E_{m}=E_{m}(0)-E_{m}(2 \pi / 3)$, plotted in Fig. 2(j).

This type of translational symmetry breaking is also relevant for, e.g., quantum discrete breathers, and has been studied extensively in this context [4]. The arguably simplest example is for the Bose-Hubbard dimer, where the quantum breather (or the "self-trapped" state) consists of a superposition of the two lowest quasidegenerate eigenstates, one being symmetric and one antisymmetric under site-permutation (a "tunneling pair"). The lifetime of the localization is given by the inverse energy splitting [6].

The eigenstate in Fig. 2(b) can be identified with the stationary dimerlike solution, noting that the three lobes in Fig. 2(b) and the black crosses in Fig. 2(a) have approximately the same positions. $\Gamma_{1}$ in Fig. 2(i) is very small, and so is the corresponding energy splitting, which means that a verylong-lived localized quantum state can be created, consisting essentially of one bright spot in a probability distribution plot. Reference [11] studied the ground state of the Bose-Hubbard Hamiltonian in more detail, however, with a positive hopping term in Eq. (1). The results in Ref. [11] are, however, connected to our work in the strong nonlinearity limit, and the reader is directed there for further details on this ground state. We note only that Fig. 2(b) is qualitatively similar to the plots in Ref. [11] for the ground state above the self-trapping transition.

The SDW solution can, on the other hand, be associated with the eigenstate in Fig. 2(f), which has bright spots at the border of the triangle, at positions corresponding to the classical SDWs (easiest to see at the bottom of the triangle). There is, however, no eigenstate with $k=2 \pi / 3$ that "matches" this eigenstate well, as can be seen both in the energy spectrum and that $\Gamma_{11}$ is rather big. The stationary SDW solution is thus more difficult to re-create quantum mechanically compared to the dimerlike solution, which is expected since the SDW is unstable and the dimerlike solution is stable. 
Looking now at Figs. 2(c)-2(g), a clear signature of the classical CFV trajectories of Figs. 2(a) is that the probability distributions are spread out over a region corresponding to where the arcs of the classical CFVs are located. The probability distributions also widen out more for increasing energy, in agreement with the increasing span of the arcs in Fig. 2(a) for higher energy. Reference [15] made a similar comparison between eigenstates and nonflipping vortices. Let us, however, emphasize that CFVs, unlike the dimerlike solution, are not stationary solutions and will therefore not correspond to a single eigenstate in each $k$ space, but rather a superposition of several. It might be tempting to identify an arc in Fig. 2(a) with an eigenstate with a similar width, but we should remember that the arc represents the whole trajectory of the CFV. Dynamically, a classical CFV is represented by a single point in Fig. 2(a), which moves back and forth along the arc. The dynamics of the corresponding quantum CFV will thus be a blob that moves in the probability distribution plot, which is a result of the interference of several eigenstates. As $N$ is increased, the extension of the bright spots will decrease. Since the total number of eigenstates also increases with $N$, and there are more eigenstates available that can contribute to the CFVs, the resulting dynamics also becomes richer, so that the blob can follow the classical point more accurately. We will see in the next section that our expectations on quantum CFVs, which have been discussed in this paragraph, are confirmed for the appropriate coherent states.

When calculating the average currents and quantum topological charge for the eigenstates, the currents are zero for $k=0$ due to symmetry. The classical topological charge would in this case be equal to zero, which the quantum topological charge sometimes is, but due to fluctuations in the cosine operator Eq. (13a), it may also take the value -1.5 . For $k=2 \pi / 3$, on the other hand, the average currents can take nonzero values. All three currents are the same, since the states are stationary, but the sign of $k$ does not indicate their direction. The quantum topological charge will generally not take the classically allowed integer values but end up in an interval either between -0.5 and 0.5 , or between \pm 1 and \pm 1.5 , this again due to quantum fluctuations. We will return to a discussion on the validity of the quantum topological charge later on.

\section{CFV COHERENT STATES}

\section{A. Dynamics}

In this section we study quantum time evolution of initial CFV coherent states: coherent states obtained by inserting a classical CFV state vector $\psi$ into Eq. (3). Consider again Fig. 1, and recall that the first column shows $\left|\psi_{j}(t)\right|^{2}$ for four classical CFVs that belong to the same continuous CFV subfamily with $\beta=1.00$. The second column of Fig. 1 displays the fully quantum time evolution of $\left\langle\hat{v}_{j}\right\rangle=\left\langle C_{0}[\psi](t)\left|\hat{v}_{j}\right| C_{0}[\psi](t)\right\rangle$, with $\psi$ the initial condition of the classical $\mathrm{CFV}$ on the same row, for $N=100$ and $\alpha=\beta /(N-1)=1 / 99$. Since the classical CFVs are nonstationary solutions, using $\psi=\psi(t)$ in Eq. (3) with different $0 \leqslant t<T$ results in different coherent states, and some effects of this will be discussed in Sec. IV C. The insets in Figs. 1(b2) and 1(c2) show $\left\langle\hat{v}_{1}\right\rangle$ and $\left\langle\hat{v}_{2}\right\rangle$ over a longer time span. The third column shows the average value of the current operators Eq. (12) while the fourth column shows the quantum topological charge, both calculated with the quantum time evolved CFV coherent state on the same row. Note that the average value of all three current operators are plotted in the third column, but that they follow each other quite closely (especially $\left\langle\hat{J}_{2}\right\rangle$ and $\left\langle\hat{J}_{3}\right\rangle$ ), and therefore are difficult to distinguish on this scale.

From these plots it is evident that the closer $\psi$ are to the dimerlike solution, the longer and more accurately do $\left\langle\hat{v}_{j}\right\rangle$ follow $\left|\psi_{j}\right|^{2}$. There is also a clear similarity in the top three rows between the oscillations in $\left\langle\hat{v}_{j}\right\rangle$ and $\left\langle\hat{J}_{j}\right\rangle$. Note though that the changes in $\left\langle\hat{v}_{j}\right\rangle$ are connected to the difference between the average currents through Eq. (11), which may be difficult to distinguish in the large plots, but is visible in the inset of Fig. 1(b3). The arguably most important aspect of the third column is, however, that the average of all three currents follow each other closely, even in the bottom row, and that currents repeatedly change direction. This is a clear signature of the $\mathrm{CFV}$, since all currents (at least their averages) are going in the same direction, and also flip at approximately the same time. The flipping is very close to periodic in the top three rows, with the exception of a period $100 \lesssim t \lesssim 150$ in Fig. 1(c3) when the oscillations in $\left\langle\hat{J}_{j}\right\rangle$ are small and rapid, and the charge flipping breaks down. This is during the time in Fig. 1(c2) when the amplitude of the oscillations in $\left\langle\hat{v}_{j}\right\rangle$ are small. It is remarkable that even in the bottom row, when $\left\langle\hat{v}_{j}\right\rangle$ deviates strongly from $\left|\psi_{j}\right|^{2}$, we may still discern a charge flipping in $\left\langle\hat{J}_{j}\right\rangle$, and that it also is somewhat regular.

Turning to the quantum topological charge in the column to the right, there is a periodic flipping between $\approx \pm 1$ for the top three rows. The exception is once again the region $100 \lesssim t \lesssim 150$ in Fig. $1(\mathrm{c} 4)$. Note though that the topological charge is generally not equal to exactly \pm 1 due to quantum fluctuations in the angles. The fluctuations in topological charge are of the order $10^{-3}$ in Figs. 1(a4) and 1(b4), while it is between $10^{-3}$ and $10^{-2}$ for Fig. 1(c4). In Fig. 1 (d4) the quantum topological charge jumps to $\approx \pm 1.5$ and $\approx \pm 0.5$ at certain points, which is mainly due to large quantum fluctuations in the cosine function Eq. (13a). We are in fact in a parameter regime where the fluctuations and uncertainty in $\cos \left(\theta_{j+1}^{(Q M)}-\theta_{j}^{(Q M)}\right)$ are large (compare again with the melting of stationary vortices in Ref. [9]), even for the top two rows, and one may argue that the angles and the quantum topological charge therefore are not well-defined quantities. We note, however, that by using this particular convention for the quantum topological charge, it does indicate the direction of the currents and thus the vortex (even in Fig. 1(d4), where the quantum topological charge takes values different from \pm 1 , the sign still indicates the currents direction).

Figure 3 shows a different perspective on the CFV coherent states' quantum time evolution, displaying the probability distribution for $\left|C_{0}[\psi](t)\right\rangle$ at different $t$ (cf. end of Sec. III). The plots marked with $(\mathrm{a} x),(\mathrm{b} x)$, and $(\mathrm{c} x)$ are the time-evolved CFV coherent states shown in Figs. 1(b2), 1(c2), and 1(d2), respectively. The time $t$ is shown in the top-right corner of each plot, and the location of the corresponding classical CFV $\psi(t)$ (with $v_{j} \rightarrow\left|\psi_{j}\right|^{2}$ ) is marked with a blue (gray) cross. 

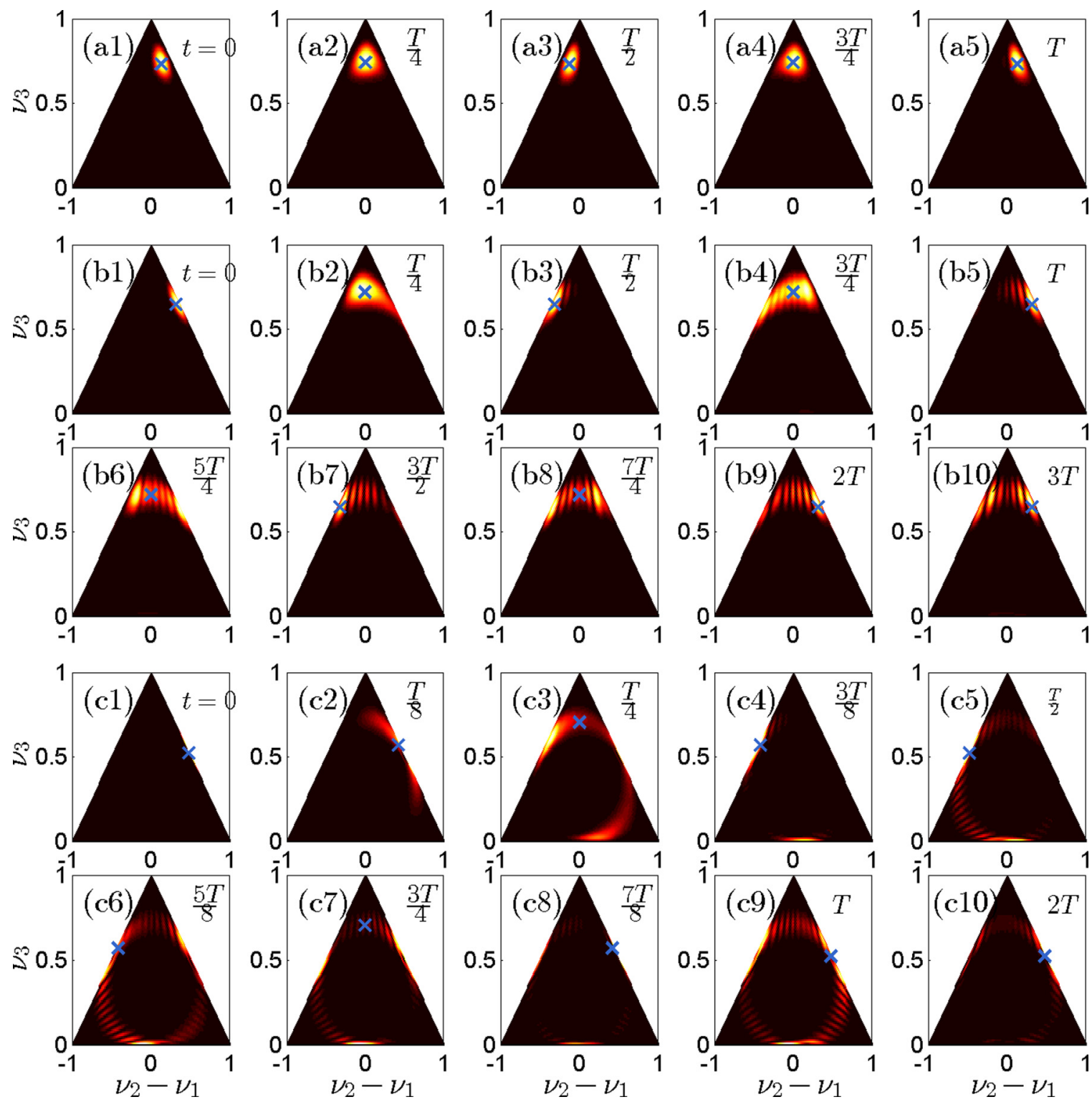

FIG. 3. Time evolution of the probability distribution $\left|\left\langle n_{1}, n_{2}, n_{3} \mid C_{0}[\psi](t)\right\rangle\right|^{2}$ of the CFV coherent states corresponding to (a1)-(a5) $\leftrightarrow$ Fig. 1(b2); (b1)-(b10) $\leftrightarrow$ Fig. 1(c2); (c1)-(c10) $\leftrightarrow$ Fig. 1(d2). The time $t$ is shown in the top-right corner of each plot. The corresponding position of the classical associated CFVs are marked with blue (gray) crosses. In (c1), (c4), and (c8) the probability distribution is localized close to the triangle border and may be difficult to distinguish.

Figures 3(a1)-3(a5) show the probability distribution over a time when the evolution of $\left\langle\hat{v}_{j}\right\rangle$ in Fig. 1(b2) follows the corresponding $\left|\psi_{j}\right|^{2}$ well. The probability distribution in Figs. 3(a1)-3(a5) therefore forms a localized bright spot that follows the classical solution well without much spreading over the time shown.

There is, however, a clear deviation between Figs. 1(c1) and 1(c2) over the time span of Figs. 3(b1)-3(b10). This deviation is also reflected in Figs. 3(b1)-3(b10), where we can see a spreading of the probability distribution over a region that corresponds to the classical trajectory. This spreading is related to the reduced amplitude for the oscillations in $\left\langle\hat{v}_{j}\right\rangle$, seen in Fig. 1(c2). The blob in the probability distribution is, however, well localized around the classical solution initially. The CFV is in some sense still preserved [which also is indicated by Fig. 1(c3)], but with quantum mechanics introducing an uncertainty about where along the trajectory of the classical $\mathrm{CFV}$ the quantum state is. The probability distribution shown in Figs. 3(a1)-3(a5) will spread out in a similar way at $t \approx 200$, as can be seen from the reduced amplitude in Fig. 1(b2). This type of spreading is oscillatory, meaning that the blob in the probability distribution alternates between spreading out and contracting, which can be seen for the amplitude modulations in the insets of Figs. 1(b2) and 1(c2). This type of spreading and contracting, and thus also the amplitude modulation of $\left\langle\hat{v}_{j}\right\rangle$, occurs in a more regular way for Fig. 1(b2) than for Fig. 1(c2), which also can be seen in the insets.

The deviation between Figs. 1(d1) and 1(d2) is almost instant, and the probability distribution is not clearly centered around the classical CFV in any of Figs. 3(c2)-3(c10). Comparing Figs. 3(c1)-3(c10) with the three top rows, we can see that there is a spreading of the probability distribution over 
the whole triangle (along a circular shape), whereas the probability distribution is restricted to the top of the triangle in the top three rows. The charge flipping is, however, not completely destroyed by this type of spreading either, which is indicated also by Fig. 1(d3), but it rather delocalizes the CFV over the trimer.

A crucial difference between the classical and quantum CFVs is that for classical CFVs the same site always is the most populated. This is also true even if the exactly periodic CFVs are slightly perturbed, since for the DNLS trimer there exist isolating KAM-tori, which strictly forbid classical spreading (cf. [33]). For the quantum CFVs, on the other hand, quantum fluctuations inevitably causes dynamical tunneling across the classical KAM-tori [36], and the probability distributions will eventually spread over the whole triangle (along a circular shape) also for the top three rows in Fig. 3, however, on a much longer timescale. For these CFV coherent states the spreading is related to the energy splitting between the $k$ spaces [cf. Fig. 2(j)].

\section{B. Projection on eigenstates}

Let us now look at how the CFV coherent states project onto the energy eigenstates. Figures 4(a) and 4(b) display $\left|\left\langle E_{m}(k) \mid C[\psi]\right\rangle\right|^{2}$ for $N=100$, where $\psi$ follows the same continuous family of classical CFVs as in Figs. 1 and 2, running from the dimerlike to the SDW solution. The configurations of all $\psi$ are chosen so that sites 1 and 3 have amplitude minima, while site 2 has a maximum, similar to the initial conditions in the first column of Fig. 1. As the CFV family is followed from the dimerlike to the SDW solution, the projection onto higher eigenstates increases, and the CFV coherent states project on several eigenstates, as was anticipated in Sec. III.

In Figs. 4(c)-4(f) we take a closer look at $\left|\left\langle C[\psi] \mid E_{m}(k)\right\rangle\right|^{2}$ for the four CFV coherent states in Fig. 1. Figure 4(c) has a large projection for $m=1$, a smaller one for $m=2$, and
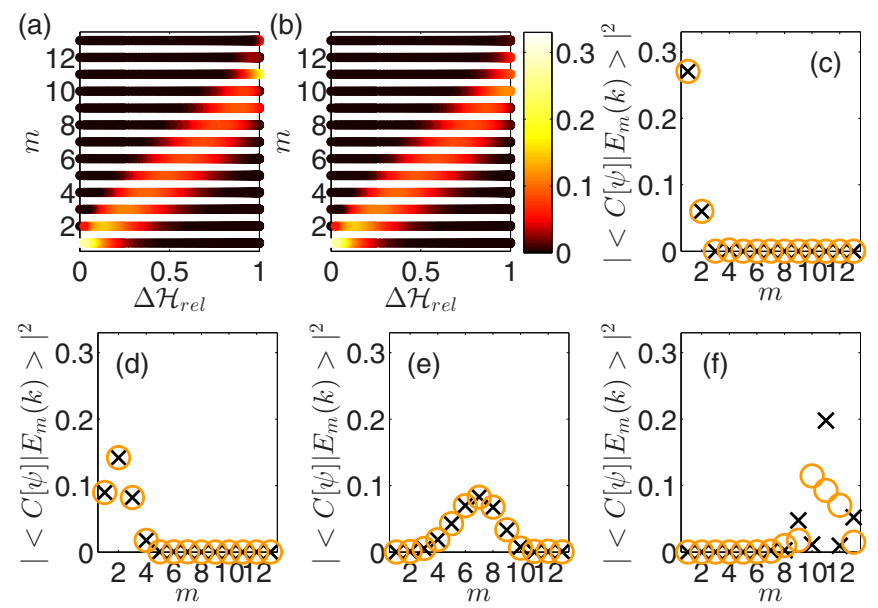

FIG. 4. $\alpha=1.00 /(N-1)$ and $N=100$. (a, b) $\left|\left\langle E_{m}(k) \mid C[\psi]\right\rangle\right|^{2}$ for the lowest eigenstates with (a) $k=0$ and (b) $k=2 \pi / 3$, with $\psi$ following the CFV subfamily with $\beta=1.00$ from the dimerlike to SDW solution. All $\psi$ are chosen so that site 1 has an amplitude minimum, similar to the initial conditions in the first column of Fig. 1. (c)-(f) show these projections in more detail for the four CFV coherent states in Figs. 1(a)-1(d), respectively, with crosses for $k=0$ and circles for $k=2 \pi / 3$. practically zero projection for $m \geqslant 3$. The frequency of the oscillations of $\left\langle\hat{v}_{j}\right\rangle$ in Fig. 1(a2) is given by $E_{2}(k)-E_{1}(k)$ [the energy splittings between the $k$ spaces for $m=1,2$ are very small in comparison, see Fig. 2(j)]. Inserting numerical values gives $2 \pi /\left(E_{2}(k)-E_{1}(k)\right) \approx 21.9$, while $T \approx 21.7$ for the $\left|\psi_{j}\right|^{2}$-oscillations in Fig. 1(a1).

In Fig. 4(d) the largest projection is no longer on the lowest eigenstates, but has moved up to $m=2$. There is also a significant population on more than two eigenstates for each $k$, namely for $m=1,2,3$. The amplitude modulation seen in Fig. 1(b2) is related to the difference between $E_{3}(k)-E_{2}(k)$ and $E_{2}(k)-E_{1}(k)$, and is thus essentially the familiar beating phenomenon, caused by the interference of two closely lying frequencies. The time period for the amplitude modulation is given by $2 \pi /\left|E_{1}(k)-2 E_{2}(k)+E_{3}(k)\right| \approx 472$, which agrees well with the inset of Fig. 1(b2). Note that the quantity $\left|E_{1}(k)-2 E_{2}(k)+E_{3}(k)\right|$ is a measure of how much these three energy levels deviate from lying on a straight line, see Fig. 2(h) (cf. the energy spectrum of the harmonic oscillator). Thus, the development of this modulation can be seen as a quantum signature of the increasing anharmonicity of the classical CFV oscillations, when moving away from the regime of linear internal-mode oscillations around the breather ground state. The time period of the classical-like oscillations in $\left\langle\hat{v}_{j}\right\rangle$ is in this case related to $4 \pi /\left(E_{3}(k)-E_{1}(k)\right) \approx 22.4$, which can be compared with $T \approx 22.6$ in Fig. 1(b1).

In Fig. 4(e) there are significant projections onto even more eigenstates than in Fig. 4(d), with the peak at $m=7$. The dynamics in Fig. 1(c2) also shows an amplitude modulation, but not in an as regular and periodic manner as in Fig. 1(b2) (compare the insets). This can again be understood as a manifestation of the beating phenomenon, but this time with more contributing frequencies. Remember also that this type of amplitude modulation is associated with a spreading of the probability distribution over a region that corresponds to the trajectory of the classical CFV, as seen in Figs. 3(b1)-3(b10).

The time evolution of all three CFV coherent states above will eventually be affected by the energy splitting between the different $k$ spaces. The probability distribution will spread out over all thirds of the triangle in plots like Fig. 3, and the values of all $\left\langle\hat{v}_{j}\right\rangle$ will thus approach $1 / 3$. The timescale for this is determined by which eigenstates that are occupied and the $k$-splitting for these [see Fig. 2(j)]

Turning now to Fig. 4(f), the dynamics of $\left\langle\hat{v}_{j}\right\rangle$ in Fig. 1(d2) shows an almost instant deviation from the corresponding $\left|\psi_{j}\right|^{2}$, and $\left\langle\hat{v}_{j}\right\rangle$ also shows a more complex time evolution compared to the other three considered CFV coherent states. The obvious difference between Fig. 4(f) and Figs. 4(c)-4(e) is that the circles do not overlap with the crosses for the populated eigenstates, meaning that the occupations of the $m$ th-lowest eigenstates with $k=0$ and $k=2 \pi / 3$, respectively, differ. This is also connected to the occupied eigenstates having a large $\Gamma_{m}$ [cf. Fig. 2(i)].

\section{CFV coherent states with other $t$}

We have up until now only considered CFV coherent states with a specific choice of $\psi$, namely $\psi=\psi(0)$, with $t=0$ set so that $\left|\psi_{1}(0)\right|^{2}$ and $\left|\psi_{3}(0)\right|^{2}$ have minima, and $\left|\psi_{2}(0)\right|^{2}$ has 

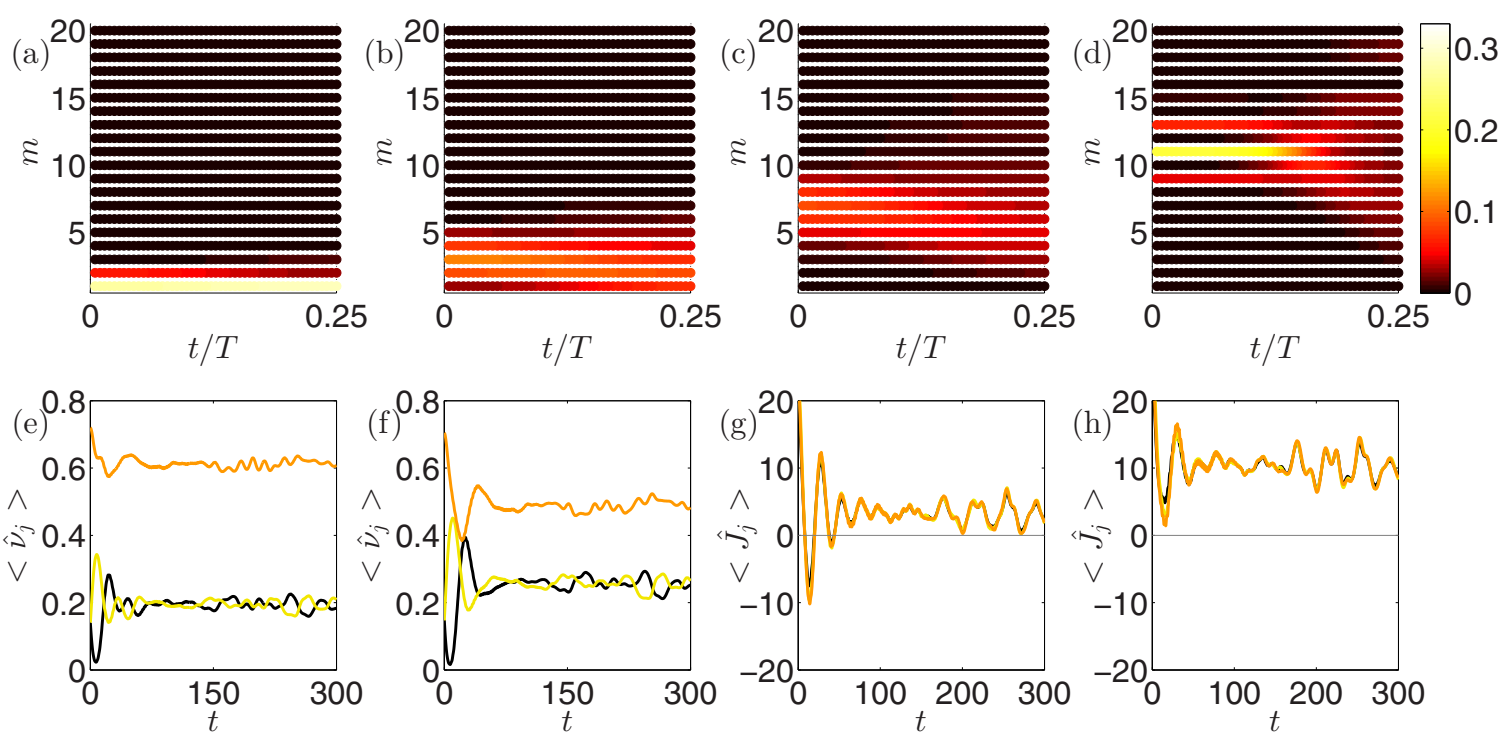

FIG. 5. $\alpha=1.00 /(N-1)$ and $N=100$. First row: $\left|\left\langle E_{m}(0) \mid C[\psi(t)]\right\rangle\right|^{2}$ for the four classical CFVs shown in Fig. 1 for different $t$, with $T$ being the period of $|\psi(t)|^{2}$. Going from left to right corresponds to going from top to bottom in Fig. 1 . The time $t$ only needs to run over one quarter of a period, because of symmetries in the classical CFVs dynamics. (e, f) Time evolution of $\left\langle\hat{v}_{j}\right\rangle$ for the CFV coherent states of $(\mathrm{c})$ and (d), respectively, with $t=T / 4$. The line coloring is the same as in Fig. 1. (g, h) Average currents, with (g) connected with (e), and (h) with (f).

a maximum. Figure 5 shows how the projection of the $\mathrm{CFV}$ coherent states onto eigenstates changes when altering $t$ of $\psi=\psi(t)$ in Eq. (3). The four plots (going from left to right) use $\psi(t)$ from the four classical CFVs in the first column in Fig. 1 (going from top to bottom). It is only necessary that the time $t$ runs from 0 to $T / 4$ in Fig. 5, due to symmetries in the classical CFVs (cf. first column of Fig. 1).

The CFV coherent states are more spread out over the eigenstates for $t \approx T / 4$ and it is around $t \approx T / 4$ that $\left|\psi_{j}\right|^{2}$ change most rapidly for the classical CFVs $\left(\left|\psi_{3}\right|^{2}\right.$ is stationary at $t=T / 4$, but changes quite rapidly in the vicinity), especially compared to $t=0$. The difference between $t=0$ and $t=T / 4$ is not so pronounced in Figs. 5(a) and 5(b), and using CFV coherent states with $\psi=\psi(T / 4)$ from these plots would result in a similar evolution of $\left\langle\hat{v}_{j}\right\rangle$ as in Figs. 1(a2) and 1(b2), respectively. For Figs. 5(c) and 5(d), on the other hand, there is a clear difference between $t=0$ and $t=T / 4$, and the time evolution of $\left\langle\hat{v}_{j}\right\rangle$ for the CFV coherent states with $\psi=\psi(T / 4)$ from these plots are shown in Figs. 5(e) and 5(f). The oscillations in $\left\langle\hat{v}_{j}\right\rangle$ decay faster, especially compared to Fig. 1(c2), and it is also difficult to discern the classical oscillations after some time. The associated average currents are plotted in Figs. 5(g) and 5(h), and even though there are oscillations in the currents, they do not change the direction. Changing $t$ in $\psi(t)$ for the CFV coherent states will thus destroy the charge flipping of the vortex for these $\psi$. Note, finally, that it is at $t=n T / 2, n \in \mathbb{Z}$, that Fig. $1(\mathrm{~d} 1)$ is closest to the configuration of a SDW solution, and also where Fig. 5(d) is most concentrated on one eigenstate.

\section{CHANGING $\boldsymbol{N}$ AND NONLINEAR PARAMETER}

We will in this section analyze how certain results from Sec. IV depend on the number of bosons $N$, focusing primarily on $N=10$ and $N=30$. Consider Fig. 6, where the top row is for $N=10$ and the bottom for $N=30$. From Figs. 6(a) and 6(b) we see that $\Gamma_{m}$ is noticeably larger than zero for all $m$ when $N=10$, and that the corresponding energy splitting is significant for $m \geqslant 2\left(\left|E_{1}(0)-E_{1}(2 \pi / 3)\right| \approx 0.02\right)$. From Figs. 6(c) and 6(d) we see that the CFV coherent states occupy mainly the three lowest eigenstates for each $k$ for $N=10$.

From the bottom row we can see that $\Gamma_{m}$, and the corresponding energy splitting, is rather small for $m<3$, while the CFV coherent states occupy the five lowest eigenstates in each $k$ space.

Consider now Fig. 7, which in the top row shows $\left\langle\hat{v}_{j}\right\rangle$ for the CFV coherent states corresponding to those in the second column of Fig. 1, but with $N=30$ (going left to right in Fig. 7 corresponds to top to bottom in Fig. 1). The second row shows the associated average currents, and the third row the CFV coherent states projection onto energy eigenstates. From this plot we can see that many of the results seen earlier for $N=100$ also hold for $N=30$. Note though the difference between Figs. 7(b3) and 4(d), where the peak is at the ground state for $N=30$ in Fig. 7(b3), but it has moved up to $m=2$ for $N=100$ in Fig. 4(d). Increasing $N$ thus "pushes" the CFV coherent state up along the eigenstates. This also means that there is a considerable occupation of three eigenstates in each $k$ space for $N=100$ in Fig. 4(d), but only of two eigenstates for $N=30$ in Fig. 7(b3). By comparing Figs. 1(b2) and $7(\mathrm{~b} 1),\left\langle\hat{v}_{j}\right\rangle$ therefore follows $\left|\psi_{j}\right|^{2}$ more closely for $N=$ 30 than $N=100$, since the "beating" amplitude modulation can only take place if there is a significant occupation of at least three eigenstates in each $k$ space, and therefore is not seen for $N=30$.

Increasing $\Delta H_{\text {rel }}$, an amplitude modulation becomes visible also for $N=30$ [Fig. 7(c1)], which is related to the significant occupation of more than two eigenstates in each $k$ space [Fig. 7(c3)]. In Figs. 7(c3) and 7(d3) we can also discern 

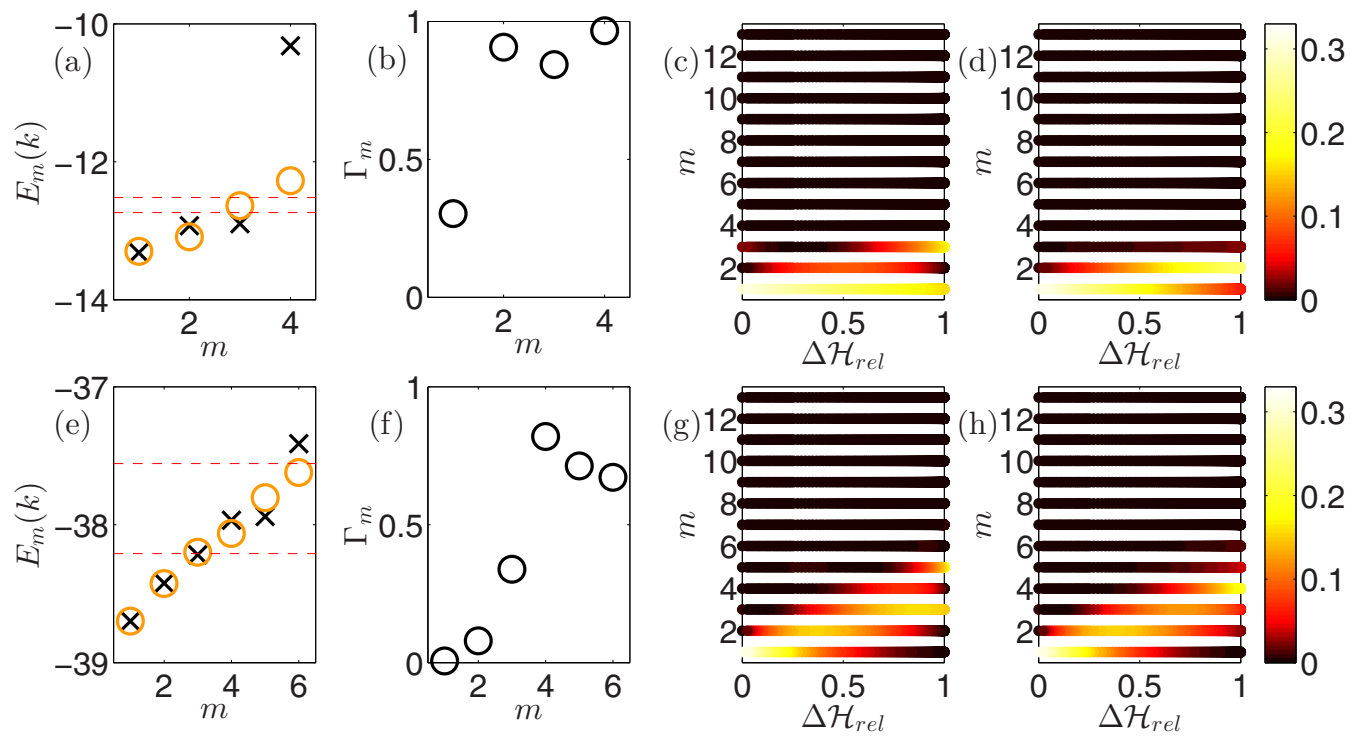

FIG. 6. $\alpha=1.00 /(N-1)$. Top row: $N=10$. Bottom row: $N=30$. (a, e) Lower part of energy spectrum for $k=0$ (crosses) and $k=2 \pi / 3$ (circles). (b, f) $\Gamma_{m}$ of Eq. (15). (c, d, g, h) Similar to Figs. 4(a) and 4(b), with (c, g) $k=0$, and (d, h) $k=2 \pi / 3$.

differences in the occupation between some of the $m$ thlowest eigenstates in either $k$ space, which is connected to nonvanishing $\Gamma_{m}$ seen in Fig. 6(f). This difference also becomes more distinct for $\psi$ closer to the unstable SDW solution, i.e., more distinct in Fig. 7(d3) than in Fig. 7(c3). Note also the difference between Figs. 7(c3) and 4(e), which are for the same $\psi$, where the circles and crosses overlap well in Fig. 4(e), but not in 7(c3). For higher $N$, we may thus follow the CFV family closer to the SDW solution before $k$-splitting becomes important.
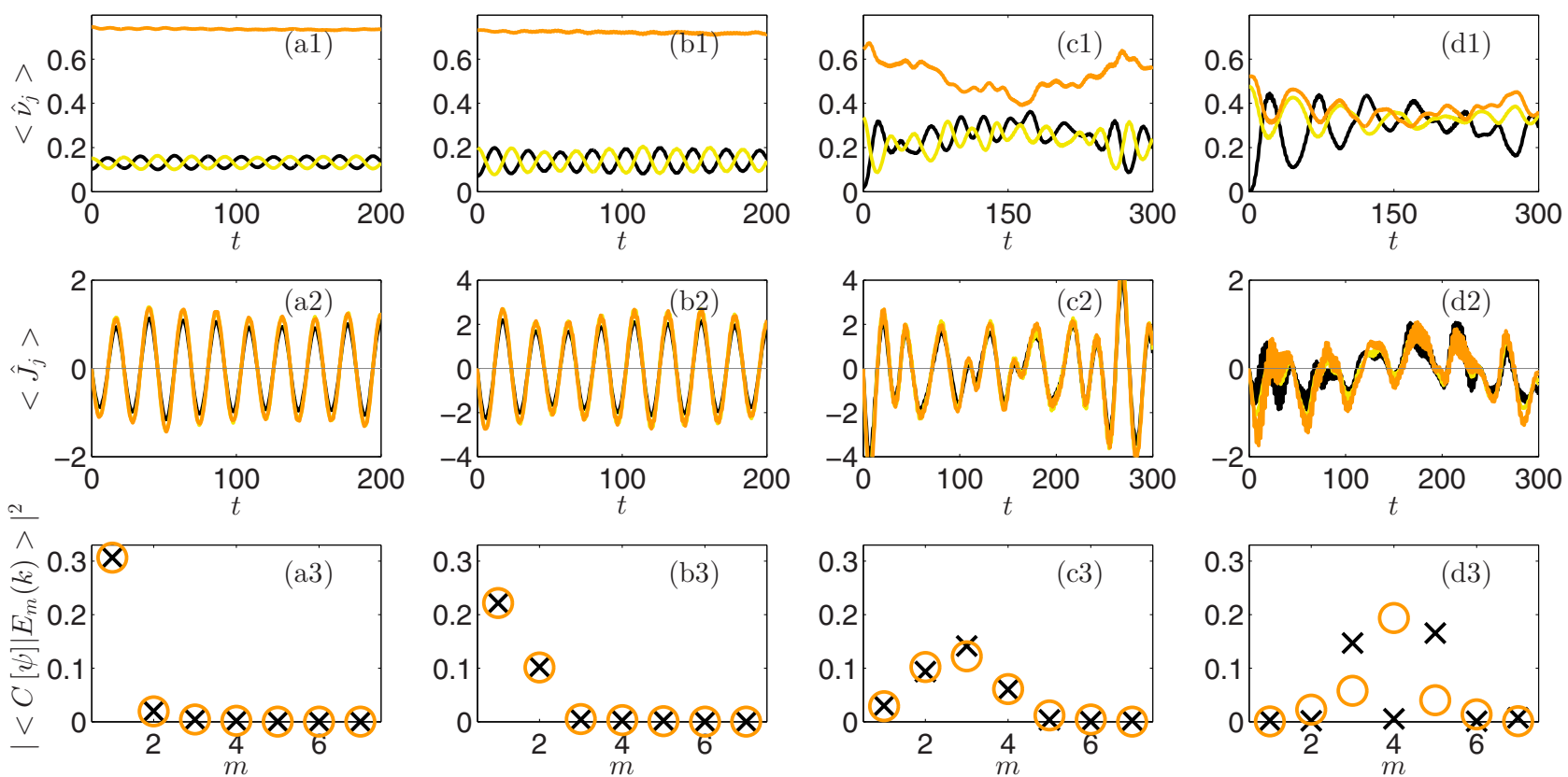

Figure 8 corresponds to Fig. 7 but with $N=10$. In this case $\Gamma_{m}$ [Fig. 6(b)] is not close to zero for any $m$, and we can see in the third row that there are noticeable differences for projections onto the $m$ th eigenstate in either $k$ space (for the occupied eigenstates). In Figs. 8(a1), 8(b1), and 8(c1) we can also see the effect of the energy splitting between the $k$ spaces, with the slow, large amplitude oscillations. $2 \pi /\left|E_{1}(0)-E_{1}(2 \pi / 3)\right| \approx 150$, which agrees well with the oscillations. Figure $9\left(\right.$ a) shows $2 \pi /\left|E_{1}(0)-E_{1}(2 \pi / 3)\right|$ as a function of $N$, and thus indicates the timescale when this

FIG. 7. $N=30, \alpha=1.00 /(N-1)$. Top row: Time evolution of $\left\langle\hat{v}_{j}\right\rangle$ for CFV coherent states corresponding the those in Fig. 1 but with $N=30$. The line coloring is the same as in Fig. 1. Second row: Associated average currents. Bottom row: Projection of CFV coherent states onto energy eigenstates. 

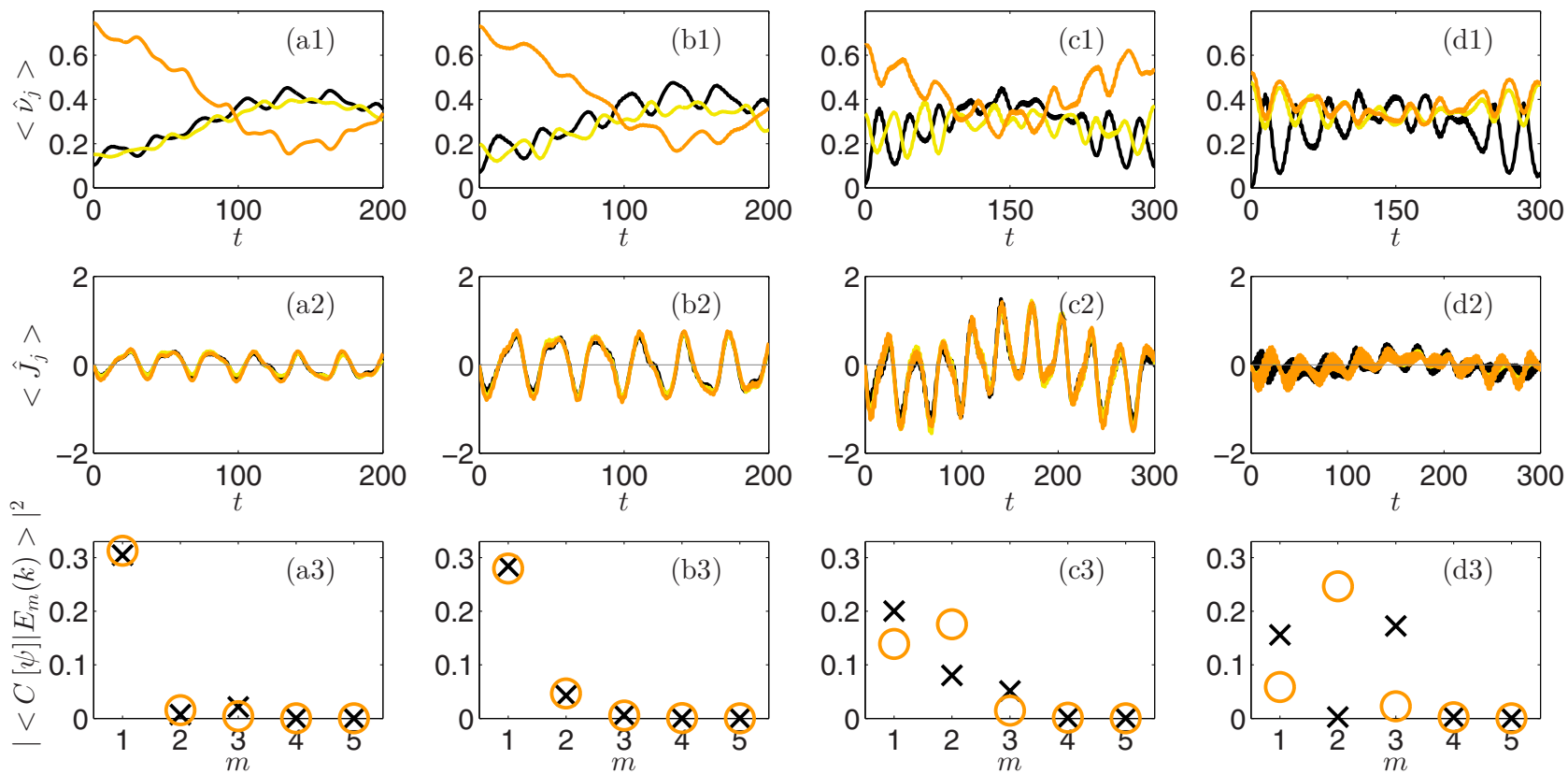

FIG. 8. Similar to Fig 7, but for $N=10$.

type of mixing becomes important, which is relevant for Figs. 1(a2), 7(a1), 8(a1), and 8(b1). This timescale is rather long already for $N=20\left(\approx 5 \times 10^{3}\right)$ compared to the time period of the classical oscillations. Figure $9($ a) also shows $2 \pi /\left|E_{m}(0)-E_{m}(2 \pi / 3)\right|$ for $m=2,3$, which come into play as these eigenstates become occupied, e.g., in Fig. 1(b2) (cf. Fig. 4). This timescale is according to Fig. 9(a) decreasing with $m$ for fixed $N$.

We can distinguish a charge flipping in the average currents for all the time evolved CFV coherent states in Figs. 7 and 8, which is more regular for $\psi$ closer to the dimerlike solution.

We have so far only considered a specific family of CFVs, which was for $\beta=1.00$ (and with $\mathcal{N}=1$ ). Most of the results
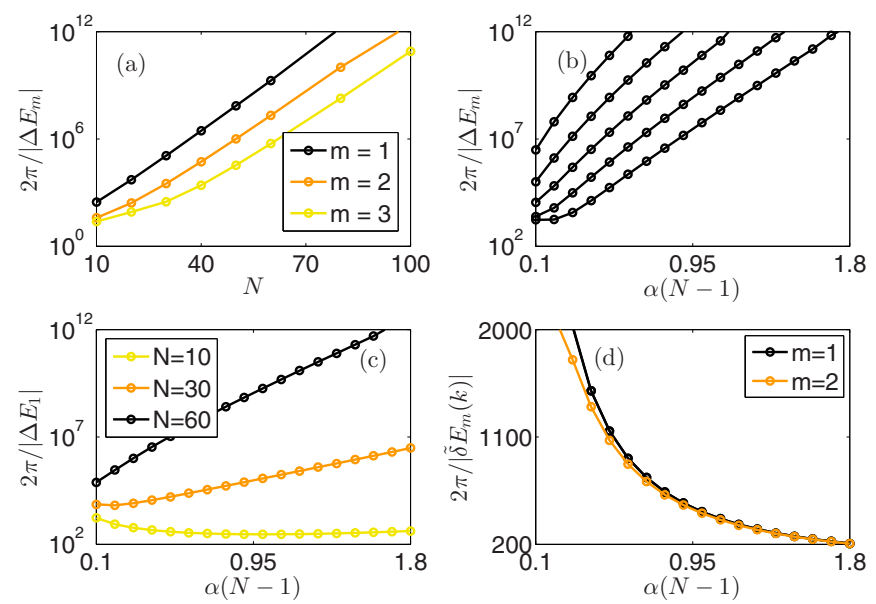

FIG. 9. (a) $2 \pi /\left|E_{m}(0)-E_{m}(2 \pi / 3)\right|$ for $\alpha=1.00 /(N-1)$. (b) $2 \pi /\left|E_{m}(0)-E_{m}(2 \pi / 3)\right|$ for (from top to bottom), $m=1,2,3,4,5$, and $N=100$. (c) $2 \pi /\left|E_{1}(0)-E_{1}(2 \pi / 3)\right|$. (d) $2 \pi / \mid E_{m}(k)-$ $2 E_{m+1}(k)+E_{m+2}(k) \mid$ for $N=100$. The curves for $k=0$ and $k=$ $2 \pi / 3$ are almost identical. that we have obtained are, however, also valid for CFV families with other $\beta$. For example, $\left\langle\hat{v}_{j}\right\rangle$ will follow the corresponding $\left|\psi_{j}\right|^{2}$ better for CFVs closer to the stable dimerlike solution, and it will also be possible to distinguish charge flipping in both the average currents and the quantum topological charge, even in the regimes where $\left\langle\hat{v}_{j}\right\rangle$ deviates strongly from $\left|\psi_{j}\right|^{2}$. A comment on this is necessary. For $\beta \gtrsim 1.8$ it is not possible to follow the CFV subfamilies (with fixed $\beta$ ) from the dimerlike all the way to the SDW solution [17]. CFVs do, however, exist in the vicinity of the dimerlike solution, and the results discussed above hold also for them.

It is thus generally possible to determine the timescale for when $\left\langle\hat{v}_{j}\right\rangle$ starts to deviate from $\left|\psi_{j}\right|^{2}$ by looking at which eigenstates that are occupied, and how their energies are related, as discussed in Sec. IV. Figures 9(b) and 9(c) show how the quantity plotted in Fig. 2(j) depends on $\alpha$ for fixed $N$, and from these figures we conclude that the timescale where the mixing of eigenstates with different $k$ becomes important is increasing with both $N$ and $\alpha(N-1)$ (at least for the plotted $N>10$ ). Figure 9(d) instead shows $2 \pi /\left|E_{m}(k)-2 E_{m+1}(k)+E_{m+2}(k)\right|$ for $N=100$, which indicates that the timescale for the amplitude modulation shown in, e.g., Fig. 1(b2) is decreasing with $\alpha$ for fixed $N$, related to the fact that a stronger nonlinearity makes classical oscillations more anharmonic (at given amplitude).

\section{SUMMARY AND CONCLUSIONS}

We have in this paper studied the Bose-Hubbard model, and searched for quantum signatures of classical CFVs from the corresponding DNLS trimer. Several such signatures have been identified, which illuminate different aspects of the quantum-classical correspondence.

Some signatures can be seen in the energy eigenstates when comparing the site amplitudes of the classical CFVs with the eigenstates' probability distributions over the different 
Fock states $\left|n_{1}, n_{2}, n_{3}\right\rangle$. This is conveniently illustrated with the triangular plots in Fig. 2 (a pedagogical advantage of the trimer), where the eigenstates' probability distributions occupy the corresponding region of the classical CFVs' trajectories.

We have furthermore considered certain CFV coherent states, and especially their dynamical properties. Quantum signatures can then be observed when comparing the evolution of the classical site amplitudes $\left|\psi_{j}\right|^{2}$ with the quantum average number of particles $\left\langle\hat{v}_{j}\right\rangle$. The classical CFVs exist in continuous families located between the linearly stable dimerlike solution (which also is the "breather" ground state) and the unstable SDW solution, and $\left\langle\hat{v}_{j}\right\rangle$ follows $\left|\psi_{j}\right|^{2}$ better for CFVs that are closer to the dimerlike solution.

We have been able to identify certain similarities and differences between $\left\langle\hat{v}_{j}\right\rangle$ and $\left|\psi_{j}\right|^{2}$ with properties of the energy spectrum, which also gives the timescales for when these deviations become important. The frequency of the classical oscillations in $\left|\psi_{j}\right|^{2}$ is related to the energy difference between adjacent energy levels of the eigenstates that the CFV coherent states occupy. For CFVs sufficiently close to the stable dimerlike solution, the classical nonlinear oscillations may to a good approximation be viewed as linear (smallamplitude and harmonic) oscillations around a nonlinear stationary state [cf. Figs. 1(a1) and 1(b1)]. Quantizing the linearized equations (Bogoliubov approximation) yields an equidistant harmonic-oscillator spectrum, and consequently in this regime the $\mathrm{CFV}$ coherent states have significant occupations only of low-lying eigenstates for which the energy differences between adjacent levels in the full Bose-Hubbard model are nearly equal (i.e., the energies $E_{m}(k)$ are almost located on a straight line when plotted against $m$, cf. Fig. 2(h)). Thus, there is a very good agreement between classical and quantum oscillation frequencies in this regime.

As the amplitude of the classical CFV oscillations increases, their nature as fully nonlinear DNLS solutions becomes evident, appearing as gradually more anharmonic with increasing oscillation period [cf. Figs. 1(c1) and 1(d1)]. A manifestation of the classical nonlinearity in the quantum model for finite $N$ is that the energies of occupied higher-energy eigenstates are no longer equidistant. The differences in these adjacent energy differences (i.e., the slight curvature seen when plotting $E_{m}(k)$ against $m$, Fig. 2(h)) cause amplitude modulations in $\left\langle\hat{v}_{j}\right\rangle$ that are not seen in the classical dynamics. Thus, in this regime the anharmonic classical oscillations with a well-defined time-period are replaced with a superposition of harmonic oscillations with several different frequencies (beating). To see these effects, it is necessary that the CFV coherent state has significant occupation of at least three eigenstates in each $k$ space.

When following a family of CFVs with increasing oscillation amplitudes from the stable dimerlike to the unstable SDW stationary solution, the CFV coherent states will eventually start to occupy eigenstates for which $\left|\left\langle E_{m}(0) \mid n_{1}, n_{2}, n_{3}\right\rangle\right|^{2}$ and $\left|\left\langle E_{m}(2 \pi / 3) \mid n_{1}, n_{2}, n_{3}\right\rangle\right|^{2}$ differ significantly. The magnitude of the CFV coherent states' projections onto the different eigenstates will also start to differ. This gives rise to additional discrepancies between the time evolution of $\left\langle\hat{v}_{j}\right\rangle$ and $\left|\psi_{j}\right|^{2}$. For larger $N$, one can follow the CFV families closer to the SDW solution before these effects become important. We remark that, in the semiclassical limit $N \rightarrow \infty$, the general relation between exact time-periodic mean-field solutions of the DNLS equation and the Fourier components of the energy spectrum of the corresponding Bose-Hubbard model can be discussed in more rigorous terms using a semiclassical trace formula [37]; however, this goes beyond the scope of the present work.

The time evolution of the coherent states' probability distribution over the Fock states has also been considered. Plotting this, the CFV coherent states form blobs in the probability distribution that follow the trajectory of the associated CFV. The different types of deviations that occur between $\left\langle\hat{v}_{j}\right\rangle$ and $\left|\psi_{j}\right|^{2}$ is related to a spreading of this blob.

The most prominent quantum CFV signatures are arguably seen in the currents and quantum topological charge of the CFV coherent states. The average currents follow each other quite closely, and also flip at approximately the same time, even in the regimes where $\left\langle\hat{v}_{j}\right\rangle$ and $\left|\psi_{j}\right|^{2}$ deviate strongly. The quantum CFVs are in this sense rather robust. Related to this is the quantum topological charge, where its sign indicates the currents direction, even though it in some regimes takes classically forbidden noninteger values. It was, however, found that for certain CFVs, close to the SDW solution, it matters which $\psi=\psi(t)$ that is used in the CFV coherent states, and that for some choices the charge flipping is destroyed.

We hope that our work will inspire experimental efforts in realizing charge flipping vortices with BECs in optical lattices, e.g., with the technique of painting potentials [16]. The realization of optical potential rings, discussed, e.g., in Ref. [38], would also provide a very promising candidate.
[1] O. Morsch and M. Oberthaler, Dynamics of Bose-Einstein condensates in optical lattices, Rev. Mod. Phys. 78, 179 (2006).

[2] I. Bloch, J. Dalibard, and W. Zwerger, Many-body physics with ultracold gases, Rev. Mod. Phys. 80, 885 (2008).

[3] A. C. Scott, J. C. Eilbeck, and H. Gilhøj, Quantum lattice solitons, Physica D 78, 194 (1994).

[4] R. A. Pinto and S. Flach, Quantum discrete breathers, in Dynamical Tunneling: Theory and Experiment, edited by S. Keshavamurthy and P. Schlagheck (CRC Press, Boca Raton, FL, 2011), Chap. 14, p. 385.

[5] J. C. Eilbeck and M. Johansson, The discrete nonlinear Schrödinger equation-20 years on, in Localization \& Energy
Transfer in Nonlinear Systems, edited by L. Vásquez, R. S. MacKay, and M. P. Zorzano (World Scientific, New Jersey, 2003), p. 44.

[6] L. Bernstein, J. C. Eilbeck, and A. C. Scott, The quantum theory of local modes in a coupled system of nonlinear oscillators, Nonlinearity 3, 293, (1990).

[7] P. Jason and M. Johansson, Exact localized eigenstates for an extended Bose-Hubbard model with pair-correlated hopping, Phys. Rev. A 85, 011603 (2012).

[8] P. Jason and M. Johansson, Quantum dynamics of lattice states with compact support in an extended Bose-Hubbard model, Phys. Rev. A 88, 033605 (2013). 
[9] C. Lee, T. J. Alexander, and Y. S. Kivshar, Melting of Discrete Vortices via Quantum Fluctuations, Phys. Rev. Lett. 97, 180408 (2006).

[10] L. J. Bernstein, Quantizing a self-trapping transition, Physica D 68, 174 (1993).

[11] P. Buonsante, V. Penna, and A. Vezzani, Quantum signatures of the self-trapping transition in attractive lattice bosons, Phys. Rev. A 82, 043615 (2010).

[12] E. Altman, A. Polkovnikov, E. Demler, B. I. Halperin, and M. D. Lukin, Superfluid-Insulator Transition in a Moving System of Interacting Bosons, Phys. Rev. Lett. 95, 020402 (2005).

[13] A. Polkovnikov, E. Altman, E. Demler, B. Halperin, and M. D. Lukin, Decay of superfluid currents in a moving system of strongly interacting bosons, Phys. Rev. A 71, 063613 (2005).

[14] P. Jason, M. Johansson, and K. Kirr, Quantum signatures of an oscillatory instability in the Bose-Hubbard trimer, Phys. Rev. E 86, 016214 (2012).

[15] G. Arwas, A. Vardi, and D. Cohen, Superfluidity and Chaos in low dimensional circuits, Scientific Rep. 5, 13433 (2015).

[16] K. Henderson, C. Ryu, C. MacCormick, and M. G. Boshier, Experimental demonstration of painting arbitrary and dynamic potentials for Bose-Einstein condensates, New J. Phys. 11, 043030 (2009).

[17] P. Jason and M. Johansson, Charge flipping vortices in the discrete nonlinear Schrödinger trimer and hexamer, Phys. Rev. E 91, 022910 (2015).

[18] L. Casetti and V. Penna, Vortex structures in a chain of coupled bosonic wells and the Mott regime, J. Low Temp. Phys. 126 455 (2002).

[19] T. J. Alexander, A. A. Sukhorukov, and Y. S. Kivshar, Asymmetric Vortex Solitons in Nonlinear Periodic Lattices, Phys. Rev. Lett. 93, 063901 (2004).

[20] A. S. Desyatnikov, M. R. Dennis, and A. Ferrando, All-optical discrete vortex switch, Phys. Rev. A 83, 063822 (2011).

[21] D. Leykam and A. S. Desyatnikov, Discrete multivortex solitons, Opt. Lett. 36, 4806 (2011).

[22] D. Leykam and A. S. Desyatnikov, Vortex switching with discrete multivortex solitons, Phys. Rev. A 86, 043812 (2012).

[23] A. Bezryadina, E. Eugenieva, and Z. Chen, Self-trapping and flipping of double-charged vortices in optically induced photonic lattices, Opt. Lett. 31, 2456 (2006).

[24] B. Terhalle, D. Göries, T. Richter, P. Rose, A. S. Desyatnikov, F. Kaiser, and C. Denz, Anisotropy-controlled topological stability of discrete vortex solitons in optically induced photonic lattices, Opt. Lett. 35, 604 (2010).
[25] F. Diebel, D. Leykam, M. Boguslawski, P. Rose, C. Denz, and A. S. Desyatnikov, All-optical switching in optically induced nonlinear waveguide couplers, Appl. Phys. Lett. 104, 26111 (2014).

[26] R. Franzosi and V. Penna, Chaotic behavior, collective modes, and self-trapping in the dynamics of three coupled Bose-Einstein condensates, Phys. Rev. E 67, 046227 (2003).

[27] J. C. Eilbeck, P. S. Lomdahl, and A. C. Scott, The discrete self-trapping equation, Physica D 16, 318 (1985).

[28] P. Buonsante, V. Penna, and A. Vezzani, Attractive ultracold bosons in a necklace optical lattice, Phys. Rev. A 72, 043620 (2005).

[29] L. Amico and V. Penna, Dynamical Mean Field Theory of the Bose-Hubbard Model, Phys. Rev. Lett. 80, 2189 (1998).

[30] D. Ellinas, M. Johansson, and P. L. Christiansen, Quantum nonlinear lattices and coherent state vectors, Physica D 134, 126 (1999).

[31] W.-M. Zhang, D. H. Feng, and R. Gilmore, Coherent states: Theory and some applications, Rev. Mod. Phys. 62, 867 (1990).

[32] M. M. Nieto, Quantum Phase and Quantum Phase Operators: Some Physics and Some History, Physica Scripta T 48, 5 (1993).

[33] M. Johansson, Hamiltonian Hopf bifurcations in the discrete nonlinear Schrödinger trimer: Oscillatory instabilities, quasiperiodic solutions and a new type of self-trapping transition, J. Phys. A: Math. Gen. 37, 2201 (2004).

[34] M. Johansson and P. Jason, Breather Mobility and the PeierlsNabarro Potential: Brief Review and Recent Progress, in Quodons in Mica-Nonlinear Localized Travelling Excitations in Crystals, edited by J. F. R. Archilla, N. Jiménez, V. J. Sánchez-Morcillo, and L. M. García-Raffi (Springer, Berlin, 2015), Chap. 6.

[35] P. Buonsante, P. G. Kevrekidis, V. Penna, and A. Vezzani, Ground-state properties of small-size nonlinear dynamical lattices, Phys. Rev. E 75, 016212 (2007).

[36] S. Keshavamurthy and P. Schlagheck (eds.), Dynamical Tunneling: Theory and Experiment (CRC Press, Boca Raton, FL, 2011).

[37] T. Engl, J. D. Urbina, and K. Richter, Periodic mean-field solutions and the spectra of discrete bosonic fields: Trace formula for the Bose-Hubbard models, Phys. Rev. E 92, 062907 (2015).

[38] L. Amico, A. Osterloh, and F. Cataliotti, Quantum Many Particle Systems in Ring-Shaped Optical Lattices, Phys. Rev. Lett. 95, 063201 (2005). 